\title{
Nonuniversal Equation of State of the Two-Dimensional Bose Gas
}

\author{
L. Salasnich \\ Dipartimento di Fisica e Astronomia “Galileo Galilei,” Università di Padova, Via Marzolo 8, 35131 Padova, Italy, \\ Consorzio Nazionale Interuniversitario per le Scienze Fisiche della Materia (CNISM), Unità di Padova, \\ Via Marzolo 8, 35131 Padova, Italy, \\ and Istituto Nazionale di Ottica (INO) del Consiglio Nazionale delle Ricerche (CNR), \\ Via Nello Carrara 1, 50019 Sesto Fiorentino, Italy \\ (Received 31 December 2016; published 29 March 2017)
}

\begin{abstract}
For a dilute two-dimensional Bose gas the universal equation of state has a logarithmic dependence on the $s$-wave scattering length. Here we derive nonuniversal corrections to this equation of state, taking account of finite-range effects of the interatomic potential. Our beyond-mean-field analytical results are obtained performing dimensional regularization of divergent zero-point quantum fluctuations within the finite-temperature formalism of functional integration. In particular, we find that in the grand canonical ensemble the pressure has a nonpolynomial dependence on the finite- range parameter and it is a highly nontrivial function of chemical potential and temperature.
\end{abstract}

DOI: 10.1103/PhysRevLett.118.130402

Introduction.-The equation of state of a uniform weakly interacting Bose gas has a long history. Universal beyond-mean-field theoretical results, which depend only on the $s$-wave scattering length $a_{s}$ of the interatomic potential, were obtained for the threedimensional (3D) bosonic system by Bogoliubov [1] and by Lee, Huang, and Yang [2,3]. In one dimension, based on a previous investigation of the 1D Bose-Fermi mapping [4], Lieb and Liniger [5] obtained the exact equation of state of a Bose gas with contact repulsive interaction. In the case of two spatial dimensions, Schick [6] found that the equation of state of a uniform 2D repulsive Bose gas contains a nontrivial logarithmic term. This remarkable result was improved by Popov [7] who obtained an equation of state which, at the leading order, reduces to Schick's one in the dilute limit (see also Refs. [8-11]). More recently, Andersen [12] and Mora and Castin [13] went one step further than Popov, finding a next-next to leading universal equation of state for the two-dimensional weakly interacting Bose gas. It is important to stress that, in the last years, various experiments with ultracold and dilute atomic gases in three dimensions [14,15] and two dimensions [16,17] have put in evidence beyond-mean-field effects on the equation of state of repulsive bosons. Moreover, experiments on 1D bosons [18,19] have shown that the Lieb-Liniger theory is needed to accurately describe the strong-coupling (i.e., low 1D density) regime.

The universal theory of the 3D weakly interacting Bose gas has been extended including corrections due to the finite range of the interatomic potential [20-27]. These corrections give a modified Gross-Pitaevskii equation [24-29] for the nonuniform condensate and nonuniversal effects for quantum fluctuations at zero temperature $[21,22]$. For a deeper understanding of the behavior of interacting bosonic systems in lower dimensionality, it is extremely important to analyze and control the nonuniversal effect induced by the finite range in the equation of state also in the case of 2D and 1D Bose gases. In this Letter we investigate finite-range effects on quantum fluctuations of a 2D Bose gas by using the finite-temperature functional integration $[30,31]$ on a local effective action. We derive the finite-temperature beyondmean-field (one-loop, Gaussian) equation of state of the bosonic system performing dimensional regularization [32] of zero-point energy. The final nonuniversal analytical result, which reduces to the universal Popov equation of state [7] in the zero-range case, exhibits a nonpolynomial dependence on the finite-range parameter.

Effective field theory for the $2 D$ Bose gas. - In the study of the 2D interacting Bose gas we adopt the path integral formalism, where the atomic bosons are described by a complex field $\psi(\mathbf{r}, \tau)$ [31]. The Euclidean Lagrangian density of the system with chemical potential $\mu$ is given by

$$
\begin{aligned}
\mathcal{L}= & \psi^{*}(\mathbf{r}, \tau)\left(\hbar \partial_{\tau}-\frac{\hbar^{2}}{2 m} \nabla^{2}-\mu\right) \psi(\mathbf{r}, \tau) \\
& +\frac{1}{2} \int d^{2} \mathbf{r}^{\prime}\left|\psi\left(\mathbf{r}^{\prime}, \tau\right)\right|^{2} V\left(\left|\mathbf{r}-\mathbf{r}^{\prime}\right|\right)|\psi(\mathbf{r}, \tau)|^{2},
\end{aligned}
$$

where $V\left(\left|\mathbf{r}-\mathbf{r}^{\prime}\right|\right)$ is the two-body interaction potential between bosons.

Given the Fourier transform $\tilde{V}(q)$ of the interaction potential $V(r)$ one can expand it at the second order in $q$ around $q=0$ finding

$$
\tilde{V}(q) \simeq g_{0}+g_{2} q^{2}=\tilde{V}_{p, 2}(q),
$$

where 


$$
g_{0}=\tilde{V}(0)=\int d^{2} \mathbf{r} V(r)
$$

and

$$
g_{2}=\frac{1}{2} \tilde{V}^{\prime \prime}(0)=-\frac{1}{4} \int d^{2} \mathbf{r} r^{2} V(r) .
$$

Thus, within this approximation where the true interatomic potential $\tilde{V}(q)$ is substituted by the pseudopotential $\tilde{V}_{p, 2}(q)$ of Eq. (2), the effective local Lagrangian density becomes

$$
\begin{aligned}
\mathcal{L}= & \psi^{*}(\mathbf{r}, \tau)\left[\hbar \partial_{\tau}-\frac{\hbar^{2}}{2 m} \nabla^{2}-\mu\right] \psi(\mathbf{r}, \tau) \\
& +\frac{g_{0}}{2}|\psi(\mathbf{r}, \tau)|^{4}-\frac{g_{2}}{2}|\psi(\mathbf{r}, \tau)|^{2}\left(\nabla^{2}|\psi(\mathbf{r}, \tau)|^{2}\right) .
\end{aligned}
$$

The term proportional to $g_{2}$ gives an improvement with respect to the contact (zero-range) approximation usually adopted in the case of ultracold and dilute atoms. In the 3D case, Gaussian (one-loop) results of Eq. (5) have been obtained in Refs. [21-23], but only at zero temperature. Here we investigate the $2 \mathrm{D}$ case, which is nontrivial also in the absence of finite range corrections, both at zero and finite temperature.

Partition function and grand potential.-The partition function $\mathcal{Z}$ of the system at temperature $T$ can then be written as [31]

$$
\mathcal{Z}=\int \mathcal{D}\left[\psi, \psi^{*}\right] \exp \left(-\frac{S\left[\psi, \psi^{*}\right]}{\hbar}\right),
$$

where

$$
S\left[\psi, \psi^{*}\right]=\int_{0}^{\hbar \beta} d \tau \int_{L^{2}} d^{2} \mathbf{r} \mathcal{L}\left(\psi, \psi^{*}\right)
$$

is the Euclidean action, $L^{2}$ is the area of the system, and $\beta \equiv 1 /\left(k_{B} T\right)$ with $k_{B}$ being Boltzmann's constant. The grand potential $\Omega$ of the system, which is a function of $\mu$ and $T$, is then obtained as [31]

$$
\Omega=-\frac{1}{\beta} \ln (\mathcal{Z}) .
$$

All the thermodynamical properties of the system can be deduced from $\Omega$ but, due to the interaction, some approximated procedure is needed to explicitly calculate $\Omega$.

The mean-field plus Gaussian (one-loop) approximation is obtained, setting

$$
\psi(\mathbf{r}, \tau)=\psi_{0}+\eta(\mathbf{r}, \tau)
$$

and expanding the action $S\left[\psi, \psi^{*}\right]$ of Eq. (7) around the uniform and constant $\psi_{0}$ up to quadratic (Gaussian) order in $\eta(\mathbf{r}, \tau)$ and $\eta^{*}(\mathbf{r}, \tau)$. In this way, taking into account Eq. (5), we find the grand potential (see also Refs. $[22,30,33])$
$\Omega\left(\mu, T, \psi_{0}\right)=\Omega_{0}\left(\mu, \psi_{0}\right)+\Omega_{g}^{(0)}\left(\mu, \psi_{0}\right)+\Omega_{g}^{(T)}\left(\mu, \psi_{0}\right)$,

where

$$
\Omega_{0}\left(\mu, \psi_{0}\right)=\left(-\mu \psi_{0}^{2}+\frac{1}{2} g_{0} \psi_{0}^{4}\right) L^{2}
$$

is the mean-field contribution (assuming a real $\psi_{0}$ ),

$$
\Omega_{g}^{(0)}\left(\mu, \psi_{0}\right)=\frac{1}{2} \sum_{\mathbf{q}} E_{\mathbf{q}}\left(\mu, \psi_{0}\right)
$$

is the zero-point energy of bosonic excitations

$$
\begin{aligned}
E_{\mathbf{q}}\left(\mu, \psi_{0}\right)= & {\left[\left(\frac{\hbar^{2} q^{2}}{2 m}-\mu+\psi_{0}^{2}\left[g_{0}+\tilde{V}_{p, 2}(q)\right]\right)^{2}\right.} \\
& \left.-\psi_{0}^{4} \tilde{V}_{p, 2}(q)^{2}\right]^{1 / 2},
\end{aligned}
$$

i.e., the zero-temperature contribution of quantum Gaussian fluctuations, while

$$
\Omega_{g}^{(T)}\left(\mu, \psi_{0}\right)=\frac{1}{\beta} \sum_{\mathbf{q}} \ln \left(1-e^{-\beta E_{\mathbf{q}}\left(\mu, \psi_{0}\right)}\right)
$$

takes into account thermal Gaussian fluctuations.

Zero-temperature results. - Imposing the crucial saddlepoint condition

$$
\frac{\partial \Omega_{0}\left(\mu, \psi_{0}\right)}{\partial \psi_{0}}=0
$$

we get

$$
\psi_{0}(\mu)=\sqrt{\frac{\mu}{g_{0}}}
$$

and the following spectrum of collective excitations:

$$
E_{q}(\mu)=\sqrt{\frac{\hbar^{2} q^{2}}{2 m}\left(\lambda(\mu) \frac{\hbar^{2} q^{2}}{2 m}+2 \mu\right)},
$$

where

$$
\lambda(\mu)=1+\frac{4 m}{\hbar^{2}} \frac{g_{2}}{g_{0}} \mu
$$

takes into account finite range effects of the interatomic potential.

By using Eq. (16) the mean-field grand potential (11) becomes

$$
\Omega_{0}(\mu)=-\frac{\mu^{2}}{2 g_{0}} .
$$

Instead, the one-loop grand potential reads 


$$
\Omega_{g}^{(0)}(\mu)=\frac{1}{2} \sum_{\mathbf{q}} E_{q}(\mu) .
$$

In the continuum limit, where $\sum_{\mathbf{q}} \rightarrow L^{2} \int d^{2} \mathbf{q} /(2 \pi)^{2}$, $\Omega_{g}^{(0)}(\mu)$ is ultraviolet divergent with $E_{q}(\mu)$ given by Eq. (17). This divergence can be regularized with dimensional regularization, where the space dimension $D$ is analytically continued $[22,32,33]$. To this end, we extend the two-dimensional integral to a generic complex $D=$ $2-\varepsilon$ dimension, and then take the limit $\varepsilon \rightarrow 0$. In this way

$$
\frac{\Omega_{g}^{(0)}}{L^{D}}=\frac{1}{2} \int \frac{d^{D} \mathbf{q}}{(2 \pi)^{D}} E_{q}=-\frac{A(\mu)}{2 \kappa^{\varepsilon}} \mu^{2} \Gamma\left(-2+\frac{1}{2} \varepsilon\right),
$$

where the regulator $\kappa$ is a crucial scale wave number which enters for dimensional reasons: $L^{D}=L^{2} \kappa^{\varepsilon}$. In Eq. (21) we have defined $A(\mu)=m /\left(2 \pi \hbar^{2} \lambda(\mu)^{3 / 2}\right)$ and $\Gamma(z)$ is the Euler gamma function, such that $\Gamma(-2+\varepsilon / 2)=1 / \varepsilon+$ $O\left(\varepsilon^{0}\right)$ for $\varepsilon \rightarrow 0$. Notice that the strengths $g_{0}$ and $g_{2}$ of the 2D Lagrangian density (5) become $g_{0} \kappa^{\varepsilon}$ and $g_{2} \kappa^{\varepsilon}$ in $D$ dimensions, but the adimensional parameter $\lambda(\mu)$ of Eq. (18) remains unchanged.

It follows that, to leading order in $1 / \varepsilon$, the Gaussian grand potential in $D$ dimensions reads

$$
\frac{\Omega_{g}^{(0)}(\mu)}{L^{D}}=-\frac{A(\mu)}{2 \varepsilon \kappa^{\varepsilon}} \mu^{2} .
$$

This expression is still divergent. Nevertheless, comparing $\Omega_{g}(\mu)$ with $\Omega_{0}(\mu)$ in $D=2-\varepsilon$ dimensions we find the total zero-temperature grand potential

$$
\frac{\Omega^{(0)}(\mu)}{L^{D}}=\frac{\Omega_{0}(\mu)}{L^{D}}+\frac{\Omega_{g}^{(0)}(\mu)}{L^{D}}=-\frac{\mu^{2}}{2 \xi_{r}(\mu, \kappa, \varepsilon)},
$$

where it appears the "running constant"

$$
\frac{1}{\xi_{r}(\mu, \kappa, \varepsilon)}=\frac{1}{g_{0} \kappa^{\varepsilon}}+\frac{A(\mu)}{\varepsilon \kappa^{\varepsilon}},
$$

which runs by changing $\kappa$ and depends on the dimension $D$ through $\varepsilon=2-D[30,32,33]$.

To remove the divergence $1 / \varepsilon$ in Eq. (24) we calculate the derivative of $1 / \xi_{r}(\mu, \kappa, \varepsilon)$ with respect to $\kappa$, finding

$$
\frac{1}{\xi_{r}(\mu, \kappa, \varepsilon)^{2}} \frac{d \xi_{r}(\mu, \kappa, \varepsilon)}{d \kappa}=\frac{\varepsilon}{g_{0} \kappa^{\varepsilon+1}}+\frac{A(\mu)}{\kappa^{\varepsilon+1}} .
$$

Now, in the limit $\varepsilon \rightarrow 0$ (i.e., $D \rightarrow 2$ ) we get

$$
\frac{1}{\xi_{r}(\mu, \kappa, 0)^{2}} \frac{d \xi_{r}(\mu, \kappa, 0)}{d \kappa}=\frac{A(\mu)}{\kappa} .
$$

This first-order differential equation can be easily solved by separation of variables, and the result is

$$
\frac{1}{\xi_{r}\left(\mu, \kappa^{\prime}, 0\right)}-\frac{1}{\xi_{r}(\mu, \kappa, 0)}=-A(\mu) \ln \left(\frac{\kappa^{\prime}}{\kappa}\right) .
$$

We set the Landau pole of Eq. (27) at the high energy scale of the system $\epsilon_{c}$; i.e., we set $1 / \xi_{r}\left(\mu, \kappa^{\prime}, 0\right)=0$ at $\kappa^{\prime}$ such that $\hbar^{2} \kappa^{\prime 2} /(2 m)=\epsilon_{c}$. Then, when $\kappa$ corresponds to the actual energy of our system, i.e., $\hbar^{2} \kappa^{2} /(2 m)=\mu$. It follows that, from Eqs. (23) with $\varepsilon \rightarrow 0$ and $A(\mu)=$ $m /\left(2 \pi \hbar^{2} \lambda(\mu)^{3 / 2}\right)$ we obtain

$$
\frac{\Omega^{(0)}(\mu)}{L^{2}}=-\frac{m}{8 \pi \hbar^{2} \lambda(\mu)^{3 / 2}} \mu^{2} \ln \left(\frac{\epsilon_{c}}{\mu}\right) .
$$

Thus, taking into account Eq. (18) and the formula $P=-\Omega / L^{2}$ that relates the pressure $P$ to the grand potential $\Omega$, we finally get the zero-temperature beyondmean-field pressure

$$
P^{(0)}(\mu)=\frac{m}{8 \pi \hbar^{2}} \frac{\mu^{2}}{(1+\chi \mu)^{3 / 2}} \ln \left(\frac{\epsilon_{c}}{\mu}\right),
$$

where

$$
\chi=\frac{4 m}{\hbar^{2}} \frac{g_{2}}{g_{0}}
$$

with $g_{0}$ given by Eq. (3) and $g_{2}$ given by Eq. (4). Moreover, following Mora and Castin [13], we set

$$
\epsilon_{c}=\frac{4 \hbar^{2}}{m a_{s}^{2} e^{2 \gamma+1 / 2}},
$$

which is the high-energy scale fixed by the 2D $s$-wave scattering length $a_{s}$, with $\gamma \simeq 0.5772$ the Euler-Mascheroni constant. Given the interatomic potential $\tilde{V}(q)$, the corresponding 2D scattering length $a_{s}$ is obtained calculating the $s$-wave phase shift $\delta_{0}(q)$ that is related to $a_{s}$ by the expression [13,34-38]

$$
\cot \left[\delta_{0}(q)\right]=\frac{2}{\pi} \ln \left(\frac{q}{2} a_{s} e^{\gamma}\right)+O\left(q^{2}\right) .
$$

In the case of contact interaction, where $\chi=0$, Eq. (29) reduces to the equation of state derived by Popov [7] from a 2D hydrodynamic Hamiltonian with $\epsilon_{c}$ an ultraviolet cutoff, which depends on the $s$-wave scattering length $a_{s}$ [39]. Moreover, using Eq. (31), one finds exactly the grand potential derived by Mora and Castin expanding the energy in powers of a small parameter [13]. Instead, if $\chi \neq 0$ Eq. (29) generalizes the zero-temperature Popov's equation of state, giving a nonpolynomial finite-range correction.

The relative difference of the pressure (29) with and without the finite-range correction is given by $\left|\left[1 /(1+\chi \mu)^{3 / 2}\right]-1\right| \simeq(3 / 2)|\chi \mu|=12 \pi n R^{2} /\left|\ln \left(n a_{s}^{2}\right)\right|$, by using $R=2 \sqrt{\left|g_{2} / g_{0}\right|}$ as characteristic range of the interatomic potential [40] and $\mu=8 \pi \hbar^{2} n /\left[m\left|\ln \left(n a_{s}^{2}\right)\right|\right]$ as leading-order chemical potential in terms of the gas 
parameter $n a_{s}^{2}$, with $n=\partial P^{(0)}(\mu) / \partial \mu$ the 2D number density $[6,7,12,13]$. Choosing, for example, $n a_{s}^{2}=10^{-5}$ and $n R^{2}=6 \times 10^{-2}$ we get a correction to the pressure of about $20 \%$ due to finite-range effects, which is much larger than the Mora-Castin next-next-to-leading universal correction [13] of about $2 \%$ for the same value of the gas parameter $n a_{s}^{2}$ [13]. This regime can be experimentally achieved with ${ }^{87} \mathrm{Rb}$ atoms, where $R=1.07 \times 10^{-2}$ micron [41], using $n=524$ atoms $/$ micron $^{2}$ and tuning the $2 \mathrm{D}$ scattering length via Feshbach resonance [42] to $a_{s}=1.38 \times 10^{-4}$ micron. In general, given a quite small gas parameter $n a_{s}^{2}$, finite-range effects become relevant for larger values of the nonuniversal adimensional parameter $n R^{2}$. In other words, sizable nonuniversal effects without next-next-to-leading universal corrections can be reached experimentally by decreasing the scattering length $a_{s}$ (through Feshbach-resonance techniques) and increasing the 2D number density $n$.

Note that, instead of using Eqs. (3) and (4), which immediately give the parameters $g_{0}$ and $g_{2}$ knowing the interatomic potential $\tilde{V}(q)$, one can alternatively establish a connection between $g_{0}$ and $g_{2}$ and familiar low-energy scattering quantities such as the $s$-wave scattering length $a_{s}$ and the $s$-wave effective range $r_{s}$ (which is not the characteristic range $R$ of the potential). In two spatial dimensions this connection is very cumbersome and highly nonlinear [34,35].

Finite-temperature results. - The finite-temperature oneloop contribution to the equation of state is obtained from Eq. (12) with Eq. (16), which gives the finite-temperature contribution

$$
P_{g}^{(T)}(\mu)=\frac{1}{4 \pi} \int_{0}^{\infty} d q q^{2} \frac{d E_{q}}{d q} \frac{1}{e^{E_{q} /\left(k_{B} T\right)}-1}
$$

to the total pressure within our Gaussian scheme. Introducing the variable $x=\beta E_{q}$, we get

$$
P_{g}^{(T)}(\mu)=-\frac{k_{B} T}{4 \pi} \int_{0}^{\infty} d x q(x, \mu, T)^{2} \frac{1}{e^{x}-1},
$$

where $q(x)$ is given by

$q(x, \mu, T)=\sqrt{\frac{2 m \mu}{\hbar^{2} \lambda(\mu)}} \sqrt{-1+\sqrt{1+\frac{\lambda(\mu)\left(k_{B} T\right)^{2} x^{2}}{\mu^{2}}}}$.

Expanding this expression at low temperature $T$, we find

$$
\begin{aligned}
P_{g}^{(T)}(\mu)= & \frac{1}{4 \pi}\left(\frac{m}{\hbar^{2}}\right)\left(k_{B} T\right)^{3}[\Gamma(3) \zeta(3) \\
& \left.-\Gamma(5) \zeta(5) \frac{\lambda(\mu)}{4 \mu^{2}}\left(k_{B} T\right)^{2}\right]
\end{aligned}
$$

where $\Gamma(x)$ is the Euler gamma function and $\lambda(\mu)$ is given by Eq. (18). Thus, we get the final grand-canonical equation of state $P(\mu, T)$, which gives the pressure as a function of both the chemical potential $\mu$ and the temperature. Explicitly,

$$
P(\mu, T)=P^{(0)}(\mu)+P_{g}^{(T)}(\mu),
$$

where $P^{(0)}(\mu)$ is given by Eq. (29) and $P_{g}^{(T)}(\mu)$ is given by Eq. (36). As clearly shown in Eq. (36), at finite temperature $T$ the role of nonuniversal effects [which are encoded into $\lambda(\mu)]$ increases as the ratio $k_{B} T / \mu$ grows. This effect is somehow expected since the details of the potential become more relevant when atoms scatter at higher energy.

Conclusions.-We have used finite-temperature oneloop functional integration to obtain the nonuniversal equation of state of a dilute and ultracold gas of bosons. We have adopted an effective field theory which includes a low-energy finite-range contribution of the interatomic interaction. The divergent zero-point energy of the system has been regularized by performing dimensional regularization. Our analytical results at zero and finite temperature are highly nontrivial generalizations of old but tricky universal formulas $[6,7,13]$ that depend only on the s-wave scattering length $a_{s}$.

The author acknowledges for partial support the BIRD project "Superfluid properties of Fermi gases in optical potentials" of the University of Padova. The author thanks Alberto Cappellaro and Flavio Toigo for enlightening discussions, and Henk Stoof for useful e suggestions.

[1] N. N. Bogoliubov, J. Phys. (Moscow) 11, 23 (1947).

[2] D. T. Lee and C. N. Yang, Phys. Rev. 105, 1119 (1957).

[3] D. T. Lee, K. Huang, and C. N. Yang, Phys. Rev. 106, 1135 (1957).

[4] M. Girardeau, J. Math. Phys. (N.Y.) 1, 516 (1960).

[5] E. H. Lieb and W. Liniger, Phys. Rev. 130, 1605 (1963).

[6] M. Schick, Phys. Rev. A 3, 1067 (1971).

[7] V. N. Popov, Theor. Math. Phys. A 11, 565 (1972).

[8] Yu. E. Lozovik and V. I. Yudson, Physica A (Amsterdam) 93A, 493 (1978).

[9] D. F. Hines, N. E. Frankel, and D. J. Mitchell, Phys. Lett. A 68, 12 (1978).

[10] D. S. Fisher and P. C. Hohenberg, Phys. Rev. B 37, 4936 (1988).

[11] E. B. Kolomeisky and J. P. Straley, Phys. Rev. B 46, 11749 (1992).

[12] J. O. Andersen, Eur. Phys. J. B 28, 389 (2002).

[13] C. Mora and Y. Castin, Phys. Rev. Lett. 102, 180404 (2009).

[14] S. B. Papp, J. M. Pino, R. J. Wild, S. Ronen, C. E. Wieman, D. S. Jin, and E. A. Cornell, Phys. Rev. Lett. 101, 135301 (2008).

[15] R. J. Wild, P. Makotyn, J. M. Pino, E. A. Cornell, and D. S. Jin, Phys. Rev. Lett. 108, 145305 (2012). 
[16] S. Nascimbene, N. Navon, F. Chevy, and C. Salomon, New J. Phys. 12, 103026 (2010).

[17] T. Yefsah, R. Desbuquois, L. Chomaz, K. J. Gunter, and J. Dalibard, Phys. Rev. Lett. 107, 130401 (2011).

[18] T. Kinoshita, T. Wenger, and D. S. Weiss, Science 305, 1125 (2004).

[19] B. Paredes, A. Widera, V. Murg, O. Mandel, S. Folling, I. Cirac, G. V. Shlyapnikov, T. W. Hansch, and I. Bloch, Nature (London) 429, 277 (2004).

[20] A. Parola, L. Salasnich, and L. Reatto, Phys. Rev. A 57, R3180 (1998).

[21] E. Braaten, H.-W. Hammer, and S. Hermans, Phys. Rev. A 63, 063609 (2001).

[22] J. O. Andersen, Rev. Mod. Phys. 76, 599 (2004).

[23] R. Roth and H. Feldmeier, Phys. Rev. A 64, 043603 (2001).

[24] H. Fu, Y. Wang, and B. Gao, Phys. Rev. A 67, 053612 (2003).

[25] J. J. Garcia-Ripoll, V. V. Konotop, B. A. Malomed, and V. M. Perez-Garcia, Math. Comput. Simul. 62, 21 (2003).

[26] A. Collin, P. Massignan, and C. J. Pethick, Phys. Rev. A 75, 013615 (2007).

[27] H. Veksler, S. Fishman, and W. Ketterle, Phys. Rev. A 90, 023620 (2014).

[28] N. T. Zinner and M. Thogersen, Phys. Rev. A 80, 023607 (2009); M. Thogersen, N. T. Zinner, and A. S. Jensen, Phys. Rev. A 80, 043625 (2009).

[29] F. Sgarlata, G. Mazzarella, and L. Salasnich, J. Phys. B 48, 115301 (2015).

[30] A. M. J. Schakel, Boulevard of Broken Symmetries (World Scientific, Singapore, 2008).

[31] A. Atland and B. Simons, Condensed Matter Field Theory (Cambridge University Press, Cambridge, England, 2006).
[32] G. 't Hooft and M. Veltman, Nucl. Phys. B44, 189 (1972).

[33] L. Salasnich and F. Toigo, Phys. Rep. 640, 1 (2016).

[34] N. N. Khuri, A. Martin, J.-M. Richard, and T. T. Wu, J. Math. Phys. (N.Y.) 50, 072105 (2009).

[35] F. Werner and Y. Castin, Phys. Rev. A 86, 013626 (2012).

[36] H. T. C. Stoof, L. P. H. de Goey, W. M. H. M. Rovers, P. S. M. Kop Jansen, and B. J. Verhaar, Phys. Rev. A 38, 1248 (1988).

[37] L.-K. Lim, C. M. Smith, and H. T. C. Stoof, Phys. Rev. A 78, 013634 (2008).

[38] M. D. Lee, S. A. Morgan, M. J. Davis, and K. Burnett, Phys. Rev. A 65, 043617 (2002)

[39] In the case of a 2D square-well potential $V(r)=-V_{0} \Theta(b-r)$, where $\Theta(x)$ is the Heaviside step function, the $s$-wave scattering length $a_{s}$ is given by $a_{s}=$ $b e^{J_{0}\left(k_{0}\right) /\left[k_{0} J_{1}\left(k_{0}\right)\right]}$ with $k_{0}=\sqrt{m V_{0} b^{2} / \hbar^{2}}$ and $J_{0}(x), J_{1}(x)$ the Bessel functions of first kind.

[40] For this characteristic range $R$, defined such that $R^{2}=4\left|g_{2} / g_{0}\right|=\left|\int d^{2} \mathbf{r} r^{2} V(r) / \int d^{2} \mathbf{r} V(r)\right|$, one finds $R=\sigma$ in the case of a 2D Gaussian potential $V(r)=V_{0} e^{-r^{2} / \sigma^{2}}$, $R=b / \sqrt{2}$ in the case of a 2D square-well potential $V(r)=-V_{0} \Theta(b-r)$, and $R=\sqrt{2} \lambda$ in the case of a $2 \mathrm{D}$ Yukawa potential $V(r)=-V_{0} e^{-r / \lambda} / r$. In all these cases the characteristic range $R$ does not depend on the strength $V_{0}$ of the interaction potential $V(r)$.

[41] J. Dalibard, in Bose-Einstein Condensation in Gases, Proceedings of the International School of Physics "Enrico Fermi," Course CXL, edited by M. Inguscio, S. Stringari, and C. Wieman (Societa Italiana di Fisica, Bologna, 1999).

[42] Z. Hadzibabic and J. Dalibard, Riv. Nuovo Cimento 34, 389 (2011). 